\title{
Parto espontáneo después de cesárea y cesá- rea de repetición en el Instituto Materno In- fantil Concepción Villaveces de Acosta año de 1958
}

\author{
Por el Personal Científico del Instituto
}

En numerosos trabajos se ha insistido en el aumento de la incidencia de la operación cesárea en los últimos años, hecho que también se ha presentado entre nosotros; sin embargo, en algunos centros obstétricos se ha exagerado su utilización, tanto en la clientela privada como en la hospitalaria, lo cual ha venido a repercutir directamente en el porvenir obstétrico de las pacientes.

Aunque son muchos los trabajos que sobre este tema se han presentado, continúa siendo motivo de discusión y por lo tanto conserva su actualidad; las causas de las diferentes opiniones son, entre otras, las diferencias de recursos hospitalarios (Banco de sangre, Laboratorio, etc.), las condiciones del personal que asiste a los servicios solicitando atención (educación, desnutrición, abandono), la escasez de control postcesárea, y por sobre todos ellos, la valoración del riesgo materno-fetal, por todos aceptado como el punto clave, pero sobreestimado por quienes sostienen que "una cesárea, siempre cesárea" y subestimado por quienes pretenden obtener gran número de partos por vía vaginal.

Por su interés, se consigna la incidencia de la operación cesárea en el Instituto Materno-Infantil, en el curso de los últimos años :

\begin{tabular}{rccc}
\multicolumn{1}{c}{ AÑO } & PARTOS & CESAREAS & PORCENTAJE \\
$1938-1943$ & 19.997 & 398 & $1.99 \%$ \\
1951 & 5.382 & 186 & 3.45 \\
1952 & 5.861 & 216 & 3.68 \\
1953 & 6.320 & 321 & 5.07 \\
1954 & 7.004 & 259 & 3.69 \\
1955 & 7.526 & 316 & 4.19 \\
1956 & 8.798 & 342 & 3.91 \\
1957 & 9.636 & 401 & 4.16 \\
1958 & 9.281 & 445 & 4.79
\end{tabular}


La primera etapa, con una incidencia tan baja (1.99\%), corresponde a una época superada; comparativamente a ella, durante los últimos seis años estudiados (1953 a 1958) la incidencia aumentó a $4.3 \%$.

Para el presente estudio fueron cuidadosamente revisadas 8.202 historias clínicas de pacientes atendidas en el Instituto en el lapso comprendido entre el $1^{\text {o }}$ de Marzo de 1958 y el 28 de $\mathrm{Fe}$ brero de 1959; se ha dividido en dos partes, considerando en la primera las pacientes con parto vaginal después de cesárea y en la segunda las pacientes con cesárea de repetición.

\section{PARTO VAGINAL DESPUES DE CESAREA}

En los diversos trabajos publicados sobre el tema $(7,8,15$, $17,22,24,25,27)$ no hay criterio unánime sobre la atención del parto de pacientes previamente operadas de cesárea; las divergencias prorienen tanto de la diferente apreciación del riesgo materno-fetal, como de las circunstancias determinadas por el medio de trabajo y condicionadas por material humano diferente.

Tres criterios principales se han planteado:

1. - El de quienes sostienen que una paciente intervenida una vez debe ser intervenida siempre, especialmente para evitar el peligro de la ruptura uterina, más frecuente durante el tratajo.

2. - El de los que permiten el parto vaginal bajo ciertas circunstancias, considerándolo peligroso cuando la intervención anterior ha sido por placenta previa, cuando la incisión ha sido clásica, cuando se han comprobado defectos de la cicatriz uterina, o cuando durante el embarazo se han presentado estados determinantes de sobredistensión uterina.

3. - El de quienes permiten el trabajo de parto a todas las pacientes con antecedente de cesárea, a excepción de aquellas en las que persiste la indicación que movió la cesárea anterior.

Castelazo-Ayala (8) sostiene que si bien la cesárea debe repetirse cuando la indicación anterior subsiste, es absolutamente inadecuado y carece de bases científicas privar de la oportunidad del parto vaginal a pacientes antes intervenidas, especialmente si han tenido partos vaginales antes o después de la cesárea. 
Permite la prueba de trabajo con las siguientes condiciones:

1. - Si la indicación de la intervención anterior no está presente.

2. - Si la cesárea anterior fue segmentaria.

3. - Si no hay antecedente de más de 2 cesáreas.

4. - Si fue intervenida por especialista y no se presentaron complicaciones operatorias trascendentes.

5. - Si el embarazo actual es normal y no hay sobre-distensión uterina

6. - Si el trabajo de parto es de evolución normal.

Sanz (17) en su estudio de pacientes con antecedente de cesárea atendidas en el Instituto Materno-Infantil durante los años 1951 y 1952, afirma que las complicaciones de estas pacientes y entre ellas la más temida, es decir, la ruptura uterina, se presentan únicamente por abandono del trabajo de parto, cuando no por seguir una conducta en total desacuerdo con los principios básicos de la obstetricia. Concluye, que las posibilidades de parto espontáneo, a término, en una paciente previamente operada de cesárea, aumentan en razón al número de partos por vía vaginal y disminuyen en razón al número de cesáreas.

Pérez-Retes (15) insiste en la importancia de la cicatriz anterior y permite la prueba de trabajo cuando no hay persistencia de la indicación anterior o sobredistensión uterina.

Muñoz y Del Corral (1) resumen la opinión de diversos autores sobre el tema y opinan que la cuidadosa evaluación de cada caso en particular dará a muchas mujeres la oportunidad de demostrar su capacidad para el parto, especialmente cuando los progresos obstétricos, el control y estudio estrictos de estas pacientes y los resultados obtenidos, así lo han demostrado.

Reyes-Ceja (24) sometió 190 pacientes con antecedente de cesárea a prueba de trabajo consistente en amniotomía en 4 a 5 centímetros de dilatación, administración de espasmolíticos, administración o no de ocitócico según la evolución del caso y vigilancia adecuada; se tomó en cuenta para la suspensión de la prueba de trabajo la inminencia de ruptura, la distocia cervical asociada, el sufrimiento fetal y la prueba de trabajo negativo, es decir, la falta de progresión de la presentación en presencia de 
buena dinámica uterina y buen estado fetal, con canal pelviano blando.

Insiste en que no debe contemporizarse con el trabajo de parto de más de 12 a 18 horas de duración, ni con la coexistencia de distocias asociadas; la aplicación baja de fórceps debe ser obligada para evitar la ruptura uterina durante el período expulsivo, e igualmente debe hacerse revisión uterina. Recomienda la aplicación de antibióticos durante el puerperio y contraindica formalmente cualquier maniobra de presión abdominal. De los casos por él atendidos solamente en 4 practicó cesárea de repetición, por sufrimiento fetal durante el trabajo; atendió 118 partos espontáneos en presentación cefálica de vértice y 4 en pelvis, aplicó 65 fórceps e hizo una versión y una embriotomía.

En los casos atendidos en el Instituto se observa tendencia a permitir el trabajo de parto a toda paciente con cesárea previa, especialmente cuando ha tenido partos por vía vaginal antes o después de ella y no hay antecedente de más de dos intervenciones; esta tendencia se ha mantenido a pesar de los graves problemas que para la atención del personal se tienen, ya que solo un porcentaje mínimo asiste a la consulta prenatal, no existe consulta postnatal para control de pacientes intervenidas y hay relativa escasez de personal en relación al elevado número de casos que ingresan solicitando los servicios de la institución. En general, todo caso ingresado con antecedente de cesárea es informado a los Jefes de Clínica, quienes después de valorarlo deciden la conducta; los distintos criterios por ellos practicados influyeron en los resultados obtenidos.

Es interesante el cuadro comparativo de partos vaginales y cesáreas de repetición, en pacientes con antecedente de cesárea, tomado de la experiencia de diversos centros obstétricos; se prefirieron los datos suministrados por escuelas o centros latinoamericanos.

Como puede apreciarse, nos encontramos en un punto intermedio entre la corriente que opera un elevado número de pacientes y la que permite el parto vaginal a gran número de ellas; por el criterio seguido en la atención del parto de pacientes con antecedente de cesárea, encajamos en el tercer grupo de los mencionados al comienzo de este trabajo. 


\begin{tabular}{lcrlrlll} 
Año & Casos & \multicolumn{2}{c}{ Parto } & \multicolumn{2}{c}{ Cesárea } & Autor & \multicolumn{1}{c}{ País } \\
1953 & 697 & 114 & $163 \%$ & 583 & $83.7 \%$ & Lane & U. S. A. \\
1955 & 141 & 26 & 18.4 & 107 & 70.8 & Pérez & Perú \\
1955 & 63 & 33 & 55.9 & 30 & 44.1 & Venezuela & Chile \\
1957 & 171 & 102 & 70.2 & 69 & 29.8 & Schmidt & Brasil \\
1957 & 123 & 85 & 69.0 & 38 & 30.0 & Riva & U. S. A. \\
1958 & 295 & 118 & 40.0 & 177 & 60.0 & Fleming & Inglaterra \\
1959 & 604 & 254 & 42.0 & 321 & 53.1 & Agüero & Venezuela \\
1958 & 221 & 124 & 56.1 & 97 & 43.9 & Instituto & Colombia
\end{tabular}

Así, al ingresar una paciente con antecedente de cesárea se plantean dos conductas:

1. La repetición de la cesárea.

2. La prueba de trabajo tendiente a obtener un parto por vía vaginal.

Con miras a ayudar a solucionar el problema planteado, $\mathrm{Mu}-$ ñoz y Del Corral (1) aconsejan practicar un detenido estudio clínico-obstétrico de toda paciente previamente operada de cesárea, considerando un aspecto quirúrgico referente a las características de la intervención y postoperatorio, un aspecto obstétrico referente a las condiciones del o de los embarazos y partos anteriores, haciendo una evaluación de la cicatriz y vigilando estrechamente el trabajo de parto, especialmente los signos de inminencia de ruptura.

\section{MATERIAL}

De las 2.21 pacientes de nuestro estudio, 124 (56.1\%) tuvieron parto vaginal; 120 de éstas tuvieron posteriormente a la cesárea practicada 239 embarazos (1.9 de promedio por paciente) de los cuales 14 fueron abortos y 225 embarazos viables.

Los 124 partos terminaron así:

Partos espontáneos

Partos intervenidos con fórceps

Partos auxiliados con ventosa

Sin dato

$\begin{array}{rr}106 & 84.4 \% \\ 12 & 10.4 \% \\ 3 & 2.6 \% \\ 3 & 2.6 \%\end{array}$

De ellos, 106 fueron de término, 10 de 8 y medio meses, 4 de 8 meses, 2 de 7 meses; no hay dato de edad del embarazo en 2 casos.

La cesárea fue practicada en el primer embarazo viable en 76 casos $(61.2 \%)$, de los cuales 27 ingresaron en trabajo de par- 
to en su segunda gestación y 49 habían tenido partos por vía vaginal después de su primera cesárea y antes de su ingreso actual.

La cesárea había sido practicada después de uno o más partos vaginales en 45 pacientes $(36.2 \%)$. Nueve pacientes $(7.2 \%)$ habían tenido dos cesáreas previas y cinco $(4 \%)$ tres intervenciones; debe anotarse que estas pacientes habían tenido partos tanto a domicilio como en el hospital, pero se observó tendencia a ser atendidas en el Instituto después de su intervención, aun cuando, como lo anotamos anteriormente no asistieran a la consulta prenatal; tal el caso de una paciente que dió a luz en su casa e ingresó en puerperio inmediato para control.

$\begin{array}{lrrrl} & \text { INDICACIONES } & \mathbf{1} & \mathbf{2} & \mathbf{3} \\ 1 & \text { Placenta previa } & 19 & & \\ 2 & \text { Presentaciones atípicas } & 16 & 1 & \\ 3 & \text { Desproporción céfalo-pélvica } & 7 & & \\ 4 & \text { Falta de encajamiento } & 6 & & \\ 5 & \text { Estrechez pélvica } & 5 & 2 & 1 \\ 6 & \text { Sufrimiento fetal } & 4 & & \\ 7 & \text { Eclampsia } & 3 & & \\ 8 & \text { Embarazo gemelar } & 3 & & \\ 9 & \text { Presentación podálica en primípara añosa } & 2 & & \\ 10 & \text { Distocia cervical } & 2 & 1 & \\ 11 & \text { Trabajo prolongado } & 1 & & \\ 12 & \text { Procidencia del cordón } & 1 & & \\ 13 & \text { Cirugía de cadera previa } & 1 & & \\ 14 & \text { Inminencia de ruptura uterina } & 1 & & 2 \\ 15 & \text { Hemorragia genital } & 1 & & \\ 16 & \text { Sin dato } & 51 & 5 & 2\end{array}$

Del estudio de las indicaciones anteriores puede colegirse que no hay unidad de criterio en los términos diagnósticos y que la incidencia de casos en los cuales no se conoció la causa de la intervención anterior fue elevado $(60 \%$ en las de 3 cesáreas anteriores, $55 \%$ en las de 2 cesáreas anteriores y $41.1 \%$ en las de 1 cesárea previa); a pesar de esto, todas las pacientes fueron sometidas a prueba de trabajo y tuvieron su parto por vía vaginal; influyó en la falta de conocimiento de la causa de las intervenciones el hecho de que algunas de estas pacientes fueron atendidas en otras instituciones y en ocasiones en medio rural, y el que 
con cierta frecuencia cambian de nombre, lo cual determina la elaboración de nuevas historias.

Incisión uterina: Fue segmentaria en 73 de los 75 casos en los cuales se encuentra dato $(97.3 \%)$ y segmento-corporal en "T" invertida en 2 casos intervenidos por situación transversa $(2.7 \%)$.

Vale la pena anotar que hay autores (2) que recomiendan el empleo de la incisión longitudinal en los casos de situación transversa, la cual favorece la extracción del feto y evita la ampliación de la incisión en forma de $\mathrm{T}$ (segmento-corporal), la cual compromete en grado mayor la circulación segmentaria y naturalmente origina un pronóstico obstétrico reservado.

Postoperatorio: Sobre 50 casos hay 15 febriles (30\%) sin que conste aclaración de la causa determinante de la fiebre.

El tiempo mediado entre la última cesárea practicada y el embarazo actual en 35 casos, es el siguiente:

\begin{tabular}{|c|c|c|}
\hline Menos & de 1 & 10 casos \\
\hline & de 1 a 2 años & 14 casos \\
\hline & de 2 a 3 años & 8 casos \\
\hline Ias & años & 3 casos \\
\hline
\end{tabular}

Causa de ingreso: Como se había anotado anteriormente, elevado número de pacientes ingresan en trabajo activo de parto y solamente un pequeño número por otras causas, entre las cuales no encontramos el antecedente de cesárea.

Trabajo de parto

Falso trabajo de parto

Hemorragia genital

Amenaza de parto prematuro

Puerperio

Afección biliar

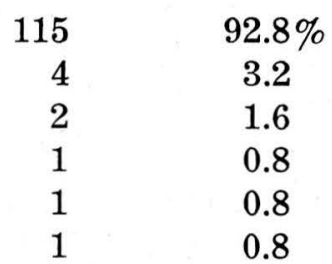

De los 115 casos ingresados en trabajo de parto 61 (53\%) tenían al examen una dilatación de 6 centímetros o más y 16 de ellos $(14 \%)$ se encontraban en dilatación completa.

Consulta prenatal: 12 de las 124 pacientes del estudio (9.7\%) asistieron a la consulta prenatal; de éstas, ninguna de las que habían sido intervenidas tres veces y solamente dos de las que tenían antecedente de 2 cesáreas. 


\begin{tabular}{|c|c|c|}
\hline dad: de 17 a 20 años & 18 & cas \\
\hline de 21 a 25 años & 35 & , \\
\hline de 26 a 30 años & 31 & " \\
\hline más de 31 años & 39 & ", \\
\hline $\begin{array}{l}\text { con fórceps } \\
\text { n ventosa }\end{array}$ & 12 & \\
\hline sic & 3 & $"$ \\
\hline
\end{tabular}

Duración del trabajo: El promedio de duración del parto fue de 4 horas 33 minutos; el trabajo más largo de 31 horas; en 5 de los casos se encontró duración mayor de 20 horas.

Acerca del trabajo en pacientes con antecedente de cesárea (1-17) se ha encontrado que éste no se realiza en forma formal posiblemente a causa de que la cicatriz uterina viene a ser una espina irritativa que transforma la dinámica, o una barrera que al trastornar la arquitectura uterina obra como un obstáculo en la repartición simétrica del estímulo contráctil; otras escuelas (24) dicen que no hay causa para el comportamiento anormal de la contractilidad. En los casos del presente estudio se encontró irregularidad en el trabajo de parto, especialmente en aquellas pacientes que habían sido intervenidas en más de una ocasión.

Revisión uterina: Solamente fue practicada en 8 casos de los 124 estudiados $(6.4 \%)$, en 4 de los cuales se había aplicado fórceps, en 2 por presencia de hemorragias de alumbramiento determinadas por retención de restos placentarios y en 2 por el antecedente de cesárea.

Todos los autores están de acuerdo en la importancia de la revisión uterina en los casos de parto vaginal con antecedente de cesárea, ya que ella permite comprobar el estado de la cicatriz y la presencia de rupturas, totales o parciales.

Es interesante uno de los casos del estudio de Agüero (7) en el cual se aplicó un fórceps laborioso y se practicó posteriormente revisión uterina; la paciente murió durante el puerperio a consecuencia de peritonitis y hemorragia, determinadas por una ruptura en la cara posterior del segmento, no encontrada durante la revisión.

Empleo de ocitócicos: En 10 pacientes se aplicó pitocin i.m. en cantidades que oscilaron entre $1 / 2$ y $2 \mathrm{U}$. generalmente en casos en que la dilatación era completa o casi completa; se aplicó 
en goteo en dextrosa al $5 \%$ en agua destilada, en 8 casos, en cantidades que variaron entre 1 y $3 \mathrm{U}$.

En estudio hecho por autores mejicanos (14) se señala que las vías subcutánea, pernasal e intramuscular deben quedar definitivamente relegadas cuando se persigue obtener efecto oxitócico de los preparados posthipofisiarios, teniendo en cuenta que la reactividad de útero a ellos, variable de una paciente a otra, está subordinada a distintos factores como la edad del embarazo, el estado del cuello, la integridad de las membranas, la paridad, el grado de distensión del útero, etc. Concluyen que de las dosis inadecuadas, las insuficiertes tienen la desventaja de no cumplir con el objetivo terapéutico que se busca y las excesivas implican riesgos materno-fetales de gran magnitud, siempre superiores a aquellos que determinaron su empleo.

Estas claras ideas acerca del empleo de los ocitócicos tienen mayor importancia cuando se trata de pacientes con antecedente de cesárea.

Se presenta el caso de una paciente atendida en el servicio, a la cual le fueron aplicados ocitócicos:

\section{HISTORIA No 51.897}

Antecedentes generales: sin importancia.

Antecedentes obstétricos: cesárea, dos partos normales con fetos muertos, dos cesáreas, dos partos intervenidos con fórceps y cuatro abortos.

Dos hospitalizaciones en el presente embarazo por amenaza de parto prematuro. Ingresa nuevamente al servicio el 14 -VI por ruptura prematura de membranas acaecida 13 horas antes.

Paciente en buen estado general, revisión de aparatos normal, tensión arterial $120 \times 80$. pulso normal. Embarazo de 34 semanas, altura uterina $28 \mathrm{cms}$. presentación cefálica, móvil; ruidos fetales positivos. Cuello posterior, corto, blando, $2 \mathrm{cms}$. de dilatación, membranas rotas.

Se aplicaron $2 \mathrm{U}$. de Pitocin en goteo, 1 ampolla de Dolantina y 1 ampolla de Penicilina; se presentaron contracciones, pero no inició parto. Al día siguiente, 15, se aplicó nuevo goteo con 3 U. de Pitocin, obteniéndose el mismo resultado. El 16 le fueron aplicados Penicilina y Foliculina. El 17 nuevo goteo con $5 \mathrm{U}$. de Pitocin, sin que se hubiera desarrollado el trabajo.

Los días 17, 18 y 19 le fueron aplicados 20 mgr. de Foliculina cada 12 horas y 1 ampolla diaria de Penicilina. El 20 se inició nueva inducción con $2 \mathrm{U}$. de Pitocin en 500 c.c. de dextrosa al $5 \%$ en agua destilada y se obtuvo la iniciación del trabajo de parto; la paciente tuvo parto intervenido con fórceps 
de desprendimiento para O.I.A con feto vivo femenino de 2.740 gramos. Salió al $4^{\circ}$ día de puerperio en buen estado general.

Reingresó 12 días después por endometritis que fue tratada a base de Bristaciclina, Ergotrate, bolsa de hielo en el abdomen, con buenos resultados.

\section{COMENTARIO}

Esta paciente con antecedente de 3 cesáreas, sin conocimiento de las causas determinantes de ellas ni detalles de las intervenciones ni postoperatorios, con ruptura prematura de membranas, fue sometida a 4 inducciones con soluciones de Pitocin, insistiéndose en su empleo a pesar de la falta de respuesta. Esta paciente bien pudo ser una más de la serie de rupturas uterinas a causa de la conducta. Tampoco le fue practicada la revisión uterina después de su parto a pesar de la gravedad de sus antecedentes.

Observaciones: En 2 pacientes con antecedente de cesárea por estrechez pélvica el parto fue igualmente espontáneo, pero los niños fueron de peso inferior al de los embarazos anteriores; una de ellas, iterativa III con feto inmaduro. En 2 pacientes con antecedente de desproporción el peso de los niños fue inferior al del embarazo anterior.

Un grupo especial está formado por las pacientes intervenidas por falta de encajamiento, 6, en las cuales se encuentran 2 con fetos de peso superior al de los embarazos anteriores, 3 con fetos de peso inferior, y 1 sin dato de él.

Muerte fetal: 8 en los 124 casos $(6.45 \%)$ promedio bajo comparado con el de otras entidades hospitalarias, entre ellas la Maternidad de Caracas $(13.3 \%)$, Jesurum $(7.1 \%)$, Castro $(10.1 \%)$, pero alto si se tienen en cuenta los promedios de otros centros especialmente americanos.

Mortalidad materna: No hubo incidencia de muertes maternas en pacientes con parto vaginal después de cesárea y a ello contribuyó la ausencia de intervenciones obstétricas difíciles como versión, fórceps complicados, etc. determinantes en otros centros de rupturas uterinas y muertes maternas.

Sobre los 221 casos de esta serie, fue permitida la prueba de trabajo a 209 y se presentaron 3 rupturas uterinas $(1.3 \%)$ en los cuales hubo supervivencia de todas las madres y solamente 
de uno de los fetos; en los tres casos la prueba estaba formalmente contraindicada.

\section{CESAREA DE REPETICION}

La distinta posición tomada por los centros hospitalarios ante la paciente con antecedente de cesárea, la presencia en estos casos de distocias asociadas o la persistencia de la causa determinante de la intervención, la diversa valoración del riesgo materno-fetal durante la prueba de trabajo, etc. han determinado un aumento de la cesárea de repetición, sistematizada en algunos centros ante un nuevo embarazo.

El mayor riesgo está determinado por la posibilidad de la ruptura uterina, cuya frecuencia, en la experiencia de algunos autores, es la siguiente:

$\begin{array}{lrcc}\text { Autor } & \text { Casos } & \text { Rupturas } & \text { Porcentaje } \\ \text { Mc Lean } & 43 & 0 & 0 \% \\ \text { Harris } & 18 & 0 & 0 \\ \text { Pauwen } & 148 & 1 & 0.6 \\ \text { Duckering } & 445 & 6 & 1.3 \\ \text { Fleming } & 150 & 2 & 1.3 \\ \text { Holland } & 448 & 18 & 4.0 \\ \text { Hindman } & 118 & 8 & 6.7 \\ \text { Instituto } & 221 & 3 & 1.3\end{array}$

Todos los autores insisten en la importancia que hay entre la ruptura propiamente dicha y la ruptura de la cicatriz anterior y proponen diversas clasificaciones para ella, siendo la más sencilla la propuesta por Eastman (33) quien las divide así:

1. Ruptura espontánea del útero intacto.

2. Ruptura traumática del útero intacto.

3. Ruptura de la cicatriz anterior.

Calcaño (6) dice que aunque en las series publicadas predomina el tipo grave de la ruptura completa en úteros intactos, son más frecuentes los casos de desunión de la cicatriz anterior la mayoría de los cuales son encontrados al revisar el útero luego del parto o con ocasión de una nueva cesárea.

Pérez-Retes (15) encuentra 7 caso de ruptura en 141 pacientes con antecedente de cesárea $(6.6 \%)$, todas ellas en pacientes 
con incisión clásica (3 de ellos durante el trabajo de parto). Arenas (4) presenta datos interesantes de los casos de ruptura uterina en la maternidad de Bucaramanga, encontrando 2 muertes maternas sobre 16 rupturas, en el grupo de pacientes con embarazos viables; en 5 de los casos $(31.2 \%)$ determinada por el empleo indiscriminado de ocitócicos.

González del Riego (16) encuentra 259 casos en la maternidad de Lima, 1 por cada 694 partos, más numerosos entre los 21 y los 30 años de edad, es decir, en la época de mayor capacidad genital; 10 fueron durante el embarazo (8 tenían antecedente de cesárea) y 249 durante el trabajo de parto, 121 de ellas espontáneas, 58 traumáticas y 70 en pacientes con antecedente de cesárea, 50 de las cuales habían sido corporales.

Rendle y Short (20) presentan 171 casos de ruptura sobre un total de 15.908 partos (una por cada 93 ) ; de ellas $38(22 \%$ ) fueron de cicatrices anteriores, 6 de las cuales eran clásicas, con una incidencia de 8 muertes maternas; 110 (64\%) fueron espontáneas y de ellas murieron 44 pacientes; 23 (14\%) fueron traumáticas con una incidencia de 11 muertes maternas. Llama la atención la elevada frecuencia de rupturas y de muertes maternas.

Cosgrove (13) en una revisión de 985 casos de muertes maternas encuentra que 72 de ellas $(7.3 \%)$ fueron determinadas por la ruptura uterina. En 30 casos (42\%) fue espontánea, en $26(36 \%)$ traumática, en $14(19 \%)$ de cicatriz anterior y en 2 $(3 \%)$ de causa desconocida. Anota la dificultad en el diagnóstico de la ruptura de cicatriz anterior, pues mientras las encontraba intactas en pacientes intervenidas por ruptura o inminencia de ella, las halló de bordes separados, asintomáticas, en pacientes sin ninguna sospecha de esta complicación, durante la revisión uterina o la cesárea de repetición. Recomienda como tratamiento de la ruptura la histerectomía.

Hay unanimidad de criterio acerca de la mayor tendencia a la ruptura de la cicatriz clásica. Sin embargo Curtis y Seidner (30) en su estudio de cesáreas clásicas practicadas en un hospital privado de los E.U.A. dicen que en ella el tiempo quirúrgico y de anestesia es menor, no hay peligro de lesiones de la vejiga y que la incidencia de adherencias, ante un buen afrontamiento, no es mayor que en la intervención segmentaria y que ante una 
buena cicatriz no hay mayor peligro de ruptura uterina; la recomiendan en caso de placenta previa y en aquellos casos especiales en que debe disminuírse el tiempo operatorio.

Debe anotarse que nuestras ideas acerca de la intervención difieren completamente de las de los autores antes mencionados, pues tanto en lo referente a tiempo de intervención y anestesia como acerca de las posibles lesiones de la vejiga y la indicación especial en caso de placenta previa, todas las solucionamos fácilmente con el empleo de la incisión segmentaria, la cual no crea el pronóstico sombrío de la intervención clásica.

Acerca de los factores predisponentes o determinantes de la desunión, varios autores $(7,9,15,22,24,29)$ han estudiado la técnica de la intervención, el tipo de postoperatorio, la paridad, el intervalo entre la intervención y el siguiente embarazo, la duración del mismo, la incidencia de sobredistensión uterina, la presencia o ausencia de trabajo, el sitio de inserción placentaria y el empleo de ocitócicos, llegando a la conclusión de que los dos únicos factores realmente importantes son, el tipo de la incisión uterina (segmentaria o corporal) y la buena calidad de la cicatriz dependiendo todo ello de la técnica operatoria y del poder de cicatrización de cada paciente.

Entre nosotros se practica el tipo de incisión propuesta por Marshall, en dos planos continuos, el primero perforante con ? ?untos cruzados y el segundo invaginante simple.

Zerpa (9) presenta un estudio de sus experiencias en 638 casos de cesáreas, tanto en primi como en multigestantes, en que practicó incisión segmentaria (2 corporo-segmentarias longitudinales) empleando sutura en un solo plano con puntos separados distantes $2 \mathrm{cms}$. el uno del otro; refiere buenos resultados en nuevos embarazos, de las pacientes así tratadas.

Sintomatología: Difiere netamente en el caso de ruptura uterina y el de ruptura de la cicatriz anterior, pues mientras en el primero hay dolor súbito e intenso en el área uterina, "shock", hipotensión, taquicardia, palidez, hipersensibilidad abdominal, hemorragia genital, desaparición de los ruidos fetales, variaciones de la presentación, interrupción del trabajo de parto, etc. en el segundo no hay manifestaciones especiales que permitan diagnosticarla, como lo habíamos anotado anteriormente, de acuerdo con la experiencia de Cosgrove. 
En los casos estudiados se encontraron 3 rupturas, ocurridas durante el trabajo de parto, cuyas historias se resumen a continuación :

\section{HISTORIA No 41.001}

Antecedentes: sin importancia.

Antecedentes obstétricos: primer embarazo ruptura fúndica al sexto mes, segundo embarazo cesárea por el antecedente de ruptura corporal.

Ingresa en su tercera gestación, a término, en buen estado general. Revisión de aparatos normal, tensión arterial $125 \times 80$. Altura uterina $30 \mathrm{cms}$. presentación cefálica alta, ruidos fetales normales. Cuello central, blando, corto y cerrado. Contracciones uterinas ocasionales, irregulares.

Un control a las 17 horas de su ingreso muestra hipertonía uterina, dolor abdominal, ruidos fetales negativos, altura uterina $26.5 \mathrm{cms}$.; pulso $102 \mathrm{al} \mathrm{mi-}$ nuto. Se aplica 1 ampolla de Dolantina i.m. y se deja en observación. Cinco horas más tarde en nuevo control, ante los mismos datos, se sospecha ruptura uterina y se interviene.

Durante la operación se encuentra una ruptura fúndica por la cual se extrae un feto muerto de 3.060 gramos; se sutura el útero en 3 planos; el postoperatorio es normal y la paciente sale del hospital a los 10 días por mejoría.

\section{COMENTARIO}

No se conocía la historia obstétrica de esta paciente o no se insistió en la investigación de la causa de sus dos intervenciones; la paciente fue dejada en el servicio sin ningún control y a las 17 horas de su ingreso, ante un cuadro que hacía presumir la complicación se aplicó un fuerte analgésico, retardando resolver unci conducta que permitía alejar el peligro de muerte a que estuvo sometida esta paciente durante crca de 5 horas. No ha debido permitirse el trabajo de parto.

\section{HISTORIA $\mathbf{N}^{0} 52.663$}

Antecedentes generales: $\sin$ importancia.

Antecedentes obstétricos: 1 embarazo de 8 meses, gemelar, complicado con eclampsia, intervenido con cesárea segmento-corporal.

Ingresa con embarazo a término, con sintomatología de toxemia, siendo tratada a base de sulfato de magnesia y Gardenal; dos días después a las 21:00 inicia trabajo de parto y 4 horas después las contracciones son regulares, cada 5 minutos, cortas y de intensidad media; ruidos fetales positivos normales.

A las 3:00 de la mañana siguiente, durante el control, se encuentra irregularidad de la superficie uterina, salida de sangre negruzca, escasa por va- 
gina; cuello largo, blando, permeable a un dedo. Se sospecha ruptura uterina.

Durante la intervención se encontró ruptura de la cicatriz anterior, con feto muerto de 2.700 gramos en la cavidad abdominal. Se practica sutura; el postoperatorio es febril; la paciente sale del hospital a los 10 días por mejoría.

\section{COMENTARIO}

No se conocían los detalles de la intervención anterior; la paciente fue controlada, aunque insuficientemente e intervenida una vez diagnosticada la ruptura; no era tributaria de prueba de trabajo esta paciente con antecedente de cesárea segmento-corporal.

\section{HISTORIA $\mathbf{N}^{\circ} \mathbf{5 8 . 4 0 8}$}

Antecedentes generales: sin importancia.

Antecedentes obstétricos: 2 cesáreas en la Clínica $1^{\circ}$ de Mayo y 2 abortos incompletos de 2 meses.

Ingresa en trabajo de parto, con embarazo a término. Buen estado general, revisión de aparatos normal, tensión arterial 120 × 80 pulso 78 al minuto. Altura uterina $28 \mathrm{cms}$. presentación cefálica alta, ruidos fetales normales; cuello borrado, blando en $4 \mathrm{cms}$. de dilatación. Diez horas más tarde es intervenida, imperativamente, poı desproporción céfalo-pélvica.

Durante la intervención se encuentra ruptura de la cicatriz anterior en una extensión de $6 \mathrm{cms}$. por donde asoma un asa de cordón pulsátil; se obtiene un feto vivo de 3.500 gramos; se practica sutura del segmento, el postoperatorio es normal y la paciente sale del hospital, a los 8 días, por mejoría.

\section{COMENTARIO}

Se desconocían la causa y el tipo de las dos cesáreas previas, a pesar de lo cual la paciente fue sometida a una prueba de trabajo larga (10 horas a partir de los $4 \mathrm{cms}$. de dilatación); no se hizo una valoración exacta del tamaño del feto; no presentó sintomatología especial que hiciera sospechar la ruptura, ya que la causa de su intervención fue desproporción; esto, confirma la opinión de varios autores, antes mencionada, acerca de la sintomatología atípica en los casos de ruptura o dehiscencia de cicatriz anterior.

\section{$M A T E R I A L$}

De las 221 pacientes $97(43.9 \%)$ fueron reintervenidas, promedio que comparamos anteriormente con el de otros centros; estas pacientes tuvieron un total de 443 embarazos (4.4 por paciente) de los cuales 25 fueron abortos y 408 embarazos viables terminados 108 por vía vaginal y 300 por cesárea. 
En 60 casos $(61.9 \%)$ las pacientes solamente tuvieron cesáreas (9 de ellas con abortos asociados) y en 37 (38.1\%) cesáreas y partos vaginales; en este segundo grupo de pacientes 6 habían sido intervenidas en su primera gestación y 31 habían tenido partos vaginales previos a la cesárea.

El número de cesáreas al ingreso era el siguiente:

\begin{tabular}{|c|c|c|c|c|}
\hline & sárea & 38 & isos & $39.1 \%$ \\
\hline 2 & $"$ & 26 & $"$ & 26.8 \\
\hline 3 & ", & 21 & " & 21.6 \\
\hline 4 & $"$ & 8 & $"$ & 8.2 \\
\hline 5 & $"$ & 3 & $"$ & 3.1 \\
\hline 6 & ", & 1 & , & 1.2 \\
\hline
\end{tabular}

El embarazo era de término en 88 casos y de 8 a 8 y medio meses en los 9 restantes.

Consulta prenatal: Solamente 15 pacientes asistieron a la consulta prenatal; $82(84.6 \%)$ ingresaron sin ningún control, la mayoría en trabajo de parto.

Causa de ingreso:

Trabajo de parto 85

Falso trabajo de parto 3

Hemorragia genital 3

Ruptura de membranas 3

Amenaza parto prematuro 2

Embarazo a término 1

Trabajo: La prueba de trabajo fue corta en 53 casos (54.6\%) es decir, de menos de 4 horas de duración; media en 11 casos $(11.3 \%)$, con duración de 4 a 8 horas; larga en 21 casos $(21.6 \%)$ con duración mayor de 8 horas; en 12 casos $(12.5 \%)$ no se permitió la prueba de trabajo y las pacientes fueron intervenidas a su ingreso. El promedio de duración del trabajo fue de 12 horas, 20 minutos, el trabajo más largo fue de 40 horas. La incidencia de la irregularidad del trabajo de parto fue mayor en este grupo de pacientes, en que había antecedente de más de una intervención. 


\begin{tabular}{|c|c|c|c|c|c|c|c|c|}
\hline & INDICACIONES & 1 & 2 & 3 & 4 & 5 & 6 & 7 \\
\hline 1 & Estrechez pélvica & 30 & 10 & 6 & 5 & 3 & & 1 \\
\hline 2 & Situación transversa & 1 & 4 & 1 & 2 & & & \\
\hline 3 & Desproporción céfalo-pélvica & 9 & 5 & 8 & 5 & 1 & & \\
\hline 4 & Eclampsia & 1 & 2 & & & & & \\
\hline 5 & Distocia cervical & 1 & 1 & 1 & & 1 & & \\
\hline 6 & Placenta previa & 2 & 2 & & & 1 & & \\
\hline 7 & Muerte habitual del feto & 2 & 2 & & & & & \\
\hline 8 & Trabajo prolongado & 2 & & 1 & & & & \\
\hline 9 & Inminencia de ruptura uterina & 1 & & & & & & \\
\hline 10 & Falta de encajamiento & 1 & 3 & & & & & \\
\hline 11 & Tumor previo & 1 & & & & & & \\
\hline 12 & Primípara añosa & 1 & & & & & & \\
\hline 13 & Procidencia del cordón & 2 & & & & & & \\
\hline 14 & Ruptura uterina & 1 & & & & & & \\
\hline 15 & Eventración & & 1 & & & & & \\
\hline 16 & Distocia ósea & & & 1 & & & & \\
\hline 17 & Cesárea anterior & & & 4 & 7 & 2 & 3 & \\
\hline 18 & Sufrimiento fetal & & 6 & 1 & 1 & & & \\
\hline 19 & Cirugía vaginal previa & & & 1 & & & & \\
\hline 20 & Presentación de frente & & 1 & & & & & \\
\hline 21 & Sin dato & 42 & 1 & 2 & 1 & & & \\
\hline
\end{tabular}

Incisión:

1 Segmentaria

2 Segmento-corporal

3 Corporal

4 Sin dato

Postoperatorio:

1 Febril

2 Afebril

3 Sin dato

Hallazgos operatorios:

Ruptura uterina

Elevación de la vejiga

Eventración

Cicatriz corporal

Hernia umbilical

Adherencias

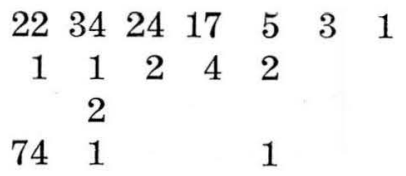

$\begin{array}{lllll}7 & 7 & 3 & 11 & 2\end{array}$

$\begin{array}{lllllll}12 & 27 & 20 & 8 & 4 & 2 & 1\end{array}$

19

\begin{tabular}{|c|c|c|}
\hline & casos & $3.1 \%$ \\
\hline & ", & 5.1 \\
\hline & $"$ & 5.1 \\
\hline & $"$ & 2.0 \\
\hline & " & 1.0 \\
\hline & $"$ & 32.9 \\
\hline
\end{tabular}


Muerte fetal: Se encontraron 13 casos (13.3\%); 2 fueron mortinatos, 2 fallecieron por neumonía, 3 por prematurez, no hay dato en 6 casos

Muerte materna: No se presentó ningún caso de muerte materna en las pacientes estudiadas en este lapso a pesar de las tres rupturas uterinas.

\section{RESUMEN Y CONCLUSIONES}

1 - Se presentan 221 casos de pacientes con antecedente de cesárea y los resultados en ellos obtenidos.

2 - Se revisan los criterios actuales para la atención del parto de estas pacientes.

3 - Puede permitirse la prueba de trabajo, bajo control riguroso, a pacientes con embarazos normales, con trabajo de parto de evolución normal, sin más de dos cesáreas previas, siempre que no persista la causa de la intervención anterior, ni se presenten distocias asociadas.

4 - Están contraindicadas las maniobras de presión abdominal y las intervenciones laboriosas.

5 - La aplicación de fórceps bajo y la revisión uterina deben ser rutinarias.

6 - El antecedente de intervención clásica es indicación de nueva cesárea.

7 - Debe establecerse una consulta postnatal para estudio y control de estas pacientes.

8 - Fueron sometidas a prueba de trabajo pacientes de las cuales se desconocían sus antecedentes, pero este es un criterio peligroso para la madre y el feto.

9 - Se practicó sutura uterina en los 3 casos de ruptura.

10 - No se practicó cirugía tendiente a la esterilización.

11 - La mortalidad fetal fue mayor en el grupo de pacientes con cesárea de repetición $(13.4 \%)$, que en el grupo de las que tuvieron parto por vía vaginal.

12 - No hubo incidencia de muertes maternas. 
13 - En las pacientes en que se presentó el parto espontáneo no hubo accidente alguno.

14 - Entre las sometidas a prueba de trabajo hubo durante la prueba 3 rupturas uterinas pero tales casos tenían antecedentes que contraindicaban la prueba.

\section{BIBLIOGRAFIA}

1 MUÑOZ-DELGADO, DEL CORRAL: Evaluación clínica para el parto después de cesárea. Rev. Ccl. Obst-Gin. X. I, 32. 1959.

2 MUÑOZ-DELGADO, СОВO-СOBO: Indicaciones selectivas de la cesárea longitudinal. Rev. Col. de Obst-Gin: XI 359. de 1960.

3 GREENHILL J. P.: Labor, operitive obstetrics. Year Book of Obst-Gyn, 1960-61.

4 ARENAS-BUENAHORA: Rupturas uterinas en la Maternidad del Hospital de Bucaramanga. Memorias II Coivención Obst-Gin: 293, 1955.

5 AGUERO OSCAR: Evaluación c.ínica de la pelvis. Rev. de Obs-Gin. de Venezuela: XVII. 377, de 1957.

6 CALCAÑO JULlo: Ruptura uterina en la Maternidad Concepción Palacios. Rev. de Obst-Gin. de Venezuela XIX 271, de 1959.

7 AGUERO, MARCANO y PAEZ - El futuro de las cesáreas. Rev. de Obst-Gin. de Venezuela: XIX.695, de 1959.

8 CASTELAZO-AYALA: Conceptos actuales sobre la operación cesárea. Rev. de ObstGin. de Venezuela: XX 1, de 1960.

9 ZERPA ELIO: La sutura del músculo uterino. Rev. de Obst-Gin. de Venezuela: $X X 127$, de 1961 .

10 GREENHILL J. P.: Principios y práctica de obstetricia de DeLee. Capítulo LXV: La operación cesárea. Edicićn 1955, Uteha.

11 GUTIERREZ PORFIRIO: Ruptura uterina. Rev. de Obst-Gin de México. XIII 332, de 1958.

12 DELGADO-CALDERON: Consideraciones sobre la operación cesárea. Tesis de grado, 1958.

13 COSGROVE ROBERT: Ruptura uterina. Rev. de Obst-Gin. de México: XV 127. de 1960.

14 ALVAREZ, CASTELAZZ SEPTIEN: Peligros del uso inadecuado de soluciones de oxitocina. Rev. de Obst-Gin de México: XV 221. de 1960.

15 PEREZ-RETES: El parto después de la operación cesárea. Rev. de Obst. del Perú. IV 55, de 1955.

16 GONZALEZ-DEL RIEGO: Ruptura uterina. Rev. de Obst-Gin. del Perú. V 36, de 1959.

17 SANZ-ARAOS: Conducta obstérrica en pacientes previamente operadas de cesárea. Rev. Col. de Obst-Gin. III 164, de 1952.

18 CORRAL-MALDONADO: Las grandes intervenciones obstétricas. Rev. Col. Obst-Gin : II 49 y 90 de 1951. 
19 ECHEVERRY PARRA: Consideraciones clínicas y estadísticas sobre la operación cesárea. Tesis de grado, Editorial Centro, 1944.

20 RENDLE, SHORT: Rupture of de gravid uterus in Uganda. Am. J. Obst-Gyn. 1114, March 1960

21 BRYANYT, RICHARD: Cesarean section in Cincinati. Am. J. Obst-Gyn: 480. March 1961.

22 MULLER, HEISER: Repeat cesarean sectiun. Am. J. Obst-Gyn: 867, May 1961.

23 MORAGUES-BERNAT: Clínica Obstétrica, capítulo LVI, 1954.

24 REYES-CEJA: La prueba de trabajo de parto en pacientes con cesárea anterior. Rev. de Obst-Gin. de México: XIV 111. de 1959.

25 BIRNBAUM, STANLEY: Postcesarean obstetrics; management of subsequent pregnancy. Obst-Gyn: Vol. 7 № 6, VI 1956.

26 ROCAMORA HECTOR: Riesgo quirúrgico de la grávida. Memoria del III Congreso Latinoamericano de Obst-Gin. México, 1958.

27 BARTOLOMEW, CALVIN: Repeat cesarean section. Obst-Gyn: vol. 7 № 2, II 1956.

28 BURKONS H.: Ruptured uterus. Obst-Gyn: vol. 7 № 6, VI 1956.

29 BACHMMANN, CULLEN: Uterire ruptured during labor. Obst-Gyn: vol. 12 № 4, $\times 1958$.

30 BAWMAN, SCHNEIDER: Classicail cesarear sections. Obst-Gyn: vol. 12 ํ. 4, X 1958.

31 POSNER, RAMIREZ: Ruptured uterus. Obst-Gyn: vol. 13 № 3, III 1959. 